\title{
Streptococcal Pharyngitis and Rheumatic Fever
}

\author{
A. Sujhithra ${ }^{1 *}\left(\mathbb{D}\right.$, S. Jayanthi ${ }^{2}\left(\mathbb{D}\right.$, M. Chokkalingam ${ }^{3}(\mathbb{C}$, \\ D. Danis Vijay ${ }^{4}\left(\mathbb{B}\right.$, R. Vidhya $^{5}\left(\mathbb{D}\right.$ and Sanjay Andrew Rajaratnam ${ }^{6}(\mathbb{C}$
}

${ }^{1}$ Department of Cardiology, Faculty of Allied Health Sciences, Chettinad Hospital and Research Institute (CHRI), Chettinad Academy of Research and Education (CARE), Kelambakkam - 603 103, Tamil Nadu, India.

${ }^{2}$ Department of Microbiology, Panimalar Medical College Hospital \& Research Institute,Varadhrajapuram, Poonamallee, Chennai - 600 123, Tamil Nadu, India.

${ }^{3}$ Department of Cardiology, Chettinad Hospital and Research Institute (CHRI), Chettinad Academy of Research and Education (CARE), Kelambakkam - 603 103, Tamil Nadu, India.

${ }^{4}$ Department of Microbiology, Karpaga Vinayaga Institute of Medical Sciences and Research Center. P.O, GST

Road, Chinna Kolambakkam, Palayanoor, Maduranthakam - 603 308, Tamil Nadu, India.

${ }^{5}$ Department of Microbiology, Chettinad Hospital and Research Institute (CHRI), Chettinad Academy of Research and Education (CARE), Kelambakkam - 603 103, Tamil Nadu, India.

${ }^{6}$ Department of Physiology, Chettinad Hospital and Research Institute (CHRI), Chettinad Academy of Research and Education (CARE), Kelambakkam - 603 103, Tamil Nadu, India.

\begin{abstract}
Streptococcus pyogenes (Group A Streptococcus) causes a variety of diseases, from benign self-limiting infections of the skin or throat to lethal infections of soft tissue accompanied by multi-organ failure. GAS is one of significant species among Gram-positive pathogens which is responsible for several suppurative infections and non-suppurative sequelae. They also cause pharyngitis, streptococcal toxic shock syndrome (STSS), necrotizing fasciitis and other diseases. Currently, global burden of RF / RHD is undervalued. In 2010, RF and RHD were estimated as 15.6 million cases and deaths around 200,000 annually. Laboratory diagnosis includes cultural techniques, serology, PYR test, Bacitracin susceptibility test and antibiotic resistance testing helps in differentiating the Streptococcus pyogenes from other groups of Streptococci. Most of the Acute Rheumatic Fever cases gets missed or does not present in the initial stage rather it has been developed into advanced Rheumatic Heart Disease condition. Modified Jones criteria in $\mathbf{2 0 1 5}$ will be helpful especially to the low risk population as it is challenging because of limited access to primary health care, diagnosis of streptococcal disease. In addition to this revised criteria, diagnosis still relies on clinical diagnostic algorithm. Vaccines based on $M$ protein and $T$ antigens are continuing to evolve with different results. Ongoing vaccine development is still challenging for the GAS research community, it will make a positive and lasting impact on the peoples globally.

Keywords: Streptococcus pyogenes, Pharyngitis, Rheumatic Fever, Pathogenesis, Vaccine strategies
\end{abstract}

*Correspondence: sujhithra.1995@gmail.com

(Received: November 24, 2021; accepted: February 1, 2022)

Citation: Sujhithra A, Jayanthi S, Chokkalingam M, Vijay DD, Vidhya R, Rajaratnam SA. Streptococcal Pharyngitis and Rheumatic Fever. J Pure App/ Microbiol. 2022;16(1):55-62. doi: 10.22207/JPAM.16.1.58

(C) The Author(s) 2022. Open Access. This article is distributed under the terms of the Creative Commons Attribution 4.0 International License which permits unrestricted use, sharing, distribution, and reproduction in any medium, provided you give appropriate credit to the original author(s) and the source, provide a link to the Creative Commons license, and indicate if changes were made. 


\section{INTRODUCTION}

Streptococcus pyogenes (Group A Streptococcus) causes a variety of diseases, from benign self-limiting infections of the skin or throat to lethal infections of soft tissue accompanied by multi-organ failure. Group A Streptococcus (GAS), a major cause of scarlet fever and it causes mortality due to sepsis and rheumatic heart disease (RHD) in industrialized countries until the arrival of the antibiotic era. ${ }^{1}$

GAS is one of significant species among Gram-positive pathogens which is responsible for several suppurative infections and nonsuppurative sequelae. ${ }^{2}$ They also cause pharyngitis, streptococcal toxic shock syndrome (STSS), necrotizing fasciitis and other diseases. It also causes acute rheumatic fever (ARF), world's leading cause of preventable heart disease in children. $^{3}$

Streptococcus pyogenes (S.pyogenes) causes ARF and RHD (rheumatic heart disease). ${ }^{4}$ ARF is due to an autoimmune response following Streptococcus pyogenes pharyngitis and throat infection. ${ }^{2}$ Rheumatic heart disease (RHD) refers to long-term heart damage caused by one or severe episodes of recurrent Streptococcal pharyngitis and ARF. It occurs about two weeks after initiation of Streptococcus infection (Gewitz et al). ${ }^{5}$

\section{Epidemiology}

Currently, global burden of RF / RHD is underestimated. In 2010, RF and RHD were estimated as 15.6 million cases and deaths around 200,000 annually. One notable aspect in the epidemiology of the disease is the discord between developed and developing countries which remains less understood with unknown reason. ${ }^{4}$

They are, however, most likely multifactorial, including variations in hygiene habits, housing differences, socioeconomic disparities, availability of antibiotics and other factors. ${ }^{4}$

This infection predominantly affects middle and low income nations, indigenous populations in developed and wealthy nations where the initial infection with S.pyogenes cannot be a treated and enables harmful post-infectious sequelae to develop (Carapetis, 2007). ${ }^{5}$ Once
ARF and RHD prevailed across all populations, widespread treatment of S.pyogenes infections and improvement in their living conditions made these diseases relatively uncommon in wealthy areas (Carapetis et al). ${ }^{1}$

India is home to $40 \%$ of all RHD-patients. In 2015, 120000 (over a third) of the 347000 deaths were caused by RHD worldwide and are estimated to have occurred in India. ${ }^{6}$

The estimated average prevalence is in the age group of 5-15 year is $0.5 / 1000$ children. In recent years, the subsequent awareness of the GAS disease and its disproportionate high burden has brought its focus on resource-limited settings, particularly in tropical regions. ${ }^{1}$

\section{Risk Factors}

Various environmental factors, age and gender are risk factors for ARF and RHD. ARF affects children aged between 5 and 14 . Recurring episodes will generally affect the older children and may occur in adolescence. Household crowding increases a likelihood of ARF, which facilitates the spread of S.pyogenes. ${ }^{5}$

\section{Classification, Structure and Antigenic Types}

Streptococci are classified depending on the serologic specificity. Serological groupings are based on differences in the antigen especially the carbohydrates in the cell wall from groups $A$ to $V$. Several structural proteins $(M, T, R)$ are found in the cell wall. $T$ and $R$ proteins in GAS serves as epidemiological markers and the $M$ proteins are virulence factors linked to resistance to phagocytosis. Based on the antigenic specificity, > 50 different types of $M$ proteins were detected and identified as virulence associated in S. pyogenes. Group-specific carbohydrates in the cell wall, $\mathrm{N}$-acetylglucosamine, M protein and peptidoglycan have the antigenic epitopes that are not only similar in their size but they even charges associated to connective tissue and also the mammalian muscle. Recent strains with the increased virulence are obviously mucoid colonies, rich in the $\mathrm{M}$ protein and encapsulated.

The capsule of S.pyogenes is made of hyaluronic acid, it is non-antigenic but chemically similar with that of the host connective tissue. The cytoplasmic membrane share the antigens which are similar to human with similar antigenic 
epitope in the human smooth, skeletal and cardiac muscles, the neuronal tissues and fibroblasts of the heart valve resulting in molecular mimicry. ${ }^{7}$

\section{Clinical Manifestation}

\section{Streptococcal Pharyngitis}

GAS pharyngitis is associated with high grade fever, increased peripheral white cell count, tonsillar exudates and tender anterior cervical lymphadenopathy. Usually these symptoms are self-limited, they resolve in 3-5 days but the suppurative infection leads to its complications like retropharyngeal and peritonsillar abscess, otitis media, suppurative lymphadenitis, meningitis and mastoiditis. ${ }^{1}$

\section{Rheumatic Fever}

ARF is characterized by fever with mono/polyarthritis, carditis (subclinical and clinical), subcutaneous nodules, chorea and erythema marginatum. ${ }^{1}$ The cardiac lesions include degeneration of heart valves and formation of inflammatory myocardial lesions called as Aschoff nodules. ${ }^{8}$

\section{Suppurative Infection \\ Streptococcal Pharyngitis}

It is the most common manifestation of GAS disease; the peak incidence is among the children of school-aged. ${ }^{1}$ It is characterized by erythema and swelling of pharyngeal mucosa with purulent exudate formation. ${ }^{8}$

\section{Pathogenesis of Streptococcal Pharyngitis}

The throat and skin are leading entry portals, after which GAS evades host defenses by expressing multiple virulence factors. ${ }^{8}$ Specific adhesion of GAS is facilitated by surface proteins. Asymptomatic colonization of the oropharynx can last for weeks. ${ }^{9}$

Pharyngitis and pyoderma are usually benign within days, with spontaneous resolution. ${ }^{1}$ Colonized individuals become susceptible to infection when they exposed to new strain or when the pharyngeal mucosa gets disrupted. ${ }^{4}$

Together with other surface proteins and hyaluronic acid capsule, $M$ protein is responsible for GAS to prevent opsonisation by obstructing complementary fixation to the bacterial cell wall. Antibodies to GAS proteins are essential in protecting against subsequent infection, particularly those that target serotype-specific epitopes of $\mathrm{M}$ protein. ${ }^{9}$

Cross-reactive antibodies in ARF were believed to develop in individuals who were genetically predisposed to GAS strains. These

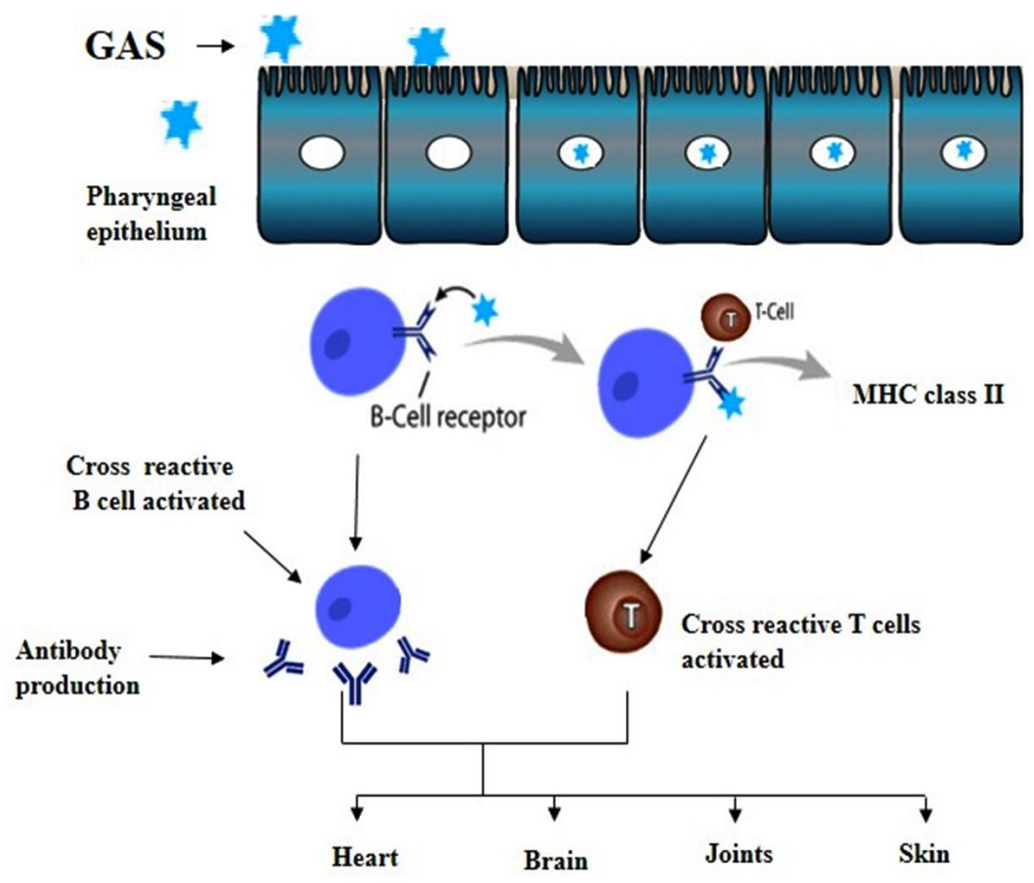

Fig. 1. Acute Rheumatic Fever-Pathogenesis. 
responses to the immune system affect the endocardial, synovial, and neural tissue leading to ARF syndrome. ${ }^{9}$

\section{Non-Suppurative Complication}

Acute rheumatic fever and acute glomerular nephritis are the non suppurative complications. ${ }^{8}$

\section{Acute Rheumatic Fever}

It occurs in people previously infected with Streptococcal sore throat or due to recurrent infection with $\mathrm{GAS}^{8}$ and the most common manifestations are painful joints and carditis. About $80 \%$ of people with ARF develops carditis affecting mitral and aortic valves predominantly. The inflammatory process takes weeks to months to resolve, but half of the affected individuals develop chronic rheumatic heart disease. Although acute rheumatic carditis does not usually result in death, chronic rheumatic heart disease is associated with a high rate of morbidity including infective endocarditis, arrhythmias, pregnancy complications, strokes and premature death. ${ }^{10}$

\section{Pathogenesis of Acute Rheumatic Fever}

Acute rheumatic fever results at a stage set by the environmental factors due to the complex interaction between GAS and the susceptible host. Joints and heart are commonly affected. Carditis can resolve or persist and develop into the continuous process of RHD.

\section{Molecular Mimicry}

There are several lines of evidence suggesting that the molecular mimicry always plays a very important role in the development of carditis by stimulating the cross reactive responses of the cellular and humoral immunity. ${ }^{5}$ The alpha-helical structures of protein are found in the $\mathrm{N}$-acetyl-beta-D-glucosamine (S.pyogenes carbohydrate antigen). M protein shares the epitopes of cross-reaction antibodies with myosin. ${ }^{11}$ Vascular cell adhesion molecule 1(VCAM 1) may be the connection between humoral and cellular immunity at the surface of the valve. VCAM 1 gets upregulated at the surface of the endothelium because of the cross reactive antibodies binding which results in the adherence of CD4+ T cells to the endothelium thereby subsequently infiltrating those cells to the valve. ${ }^{5}$ Laboratory Diagnosis Clinical Specimens

Collection of a well-taken throat swab is the success of isolating GAS in culture. Streptococcus species can be identified from the throat swab by culture. Specimens should be collected and processed in a laboratory before initiating antibiotic treatment. ${ }^{12,13}$

\section{Microscopy}

Gram staining of pus or wound swab shows pus cells and Gram positive cocci in short chains $(0.5-1 \mu \mathrm{m}) .^{8}$

\section{Cultural Identification}

Laboratory diagnosis of streptococcal pharyngitis depends on $\beta$-hemolytic streptococci being successfully isolated and identified. ${ }^{12}$ Since Streptococcus pyogenes are fastidious, enriched media like blood, carbohydrate or serum are used to isolate them. In Blood agar plates, small colonies measuring $0.5-1 \mathrm{~mm}$, circular, pin point, semitransparent, low convex with a wide zone of beta hemolysis. ${ }^{8}$

As Streptococcus pyogenes is a gram positive bacteria, providing adequate conditions and selective media (agar media containing phenylethyl alcohol or Columbia agar with nalidixic acid and colistin) for culturing these organisms. ${ }^{14}$ For culturing throat swabs, 5\% sheep blood agar should be used and incubated it for 24 hours. It is the gold standard method for diagnosing S.pyogenes in case of acute pharyngitis. ${ }^{15}$ After 24 hours of incubation at $35-37^{\circ} \mathrm{C}$, ample growth of colonies will be observed in most of the cases of acute pharyngitis. Instead of ample growth, if few colonies will be seen, then the diagnosis will be difficult and even these patients were considered as carriers rather than the acute cases. ${ }^{16}$ Followed by, the cultures should be tested for PYR and bacitracin sensitive test for the presumptive identification of S.pyogenes. A positive test of Lancefield group A antigen test will be considered as definitive diagnosis of S.pyogenes. A total of 48 hours will be taken for confirming the negative results. ${ }^{14}$

\section{Pyrrolidonyl aryl amidase Test (PYR)}

It is used for the differentiation of S. pyogenes from other $\beta$-hemolytic groups of streptococci which contains the similar morphology and also tests for the presence of pyrrolidonyl aminopeptidase enzyme. Addition of cinnamaldehyde reagent to this enzyme will hydrolyzes L-pyrrolidonyl- $\beta$-naphthylamide (PYR) into $\beta$-naphthylamide, results in producing red 
color. To avoid misidentification, we have to distinguish Streptococcus from Enterococcus before PYR testing. This test should be performed on pure culture in order to avoid the false results caused by other PYR positive strains which is present in mixed cultures. ${ }^{14,17}$

\section{Bacitracin susceptibility}

It is the method of choice for GAS identification in many laboratories. This test has a sensitivity of $>95 \%$ and it is not recommended to perform alone since streptococci group $\mathrm{G}$ and $\mathrm{C}$ may give the false-positive results. ${ }^{18,19}$

Streptococcus pyogenes can be differentiated from other non-group $A \beta$-hemolytic streptococci by their increased sensitivity to bacitracin. The increased sensitivity to bacitracin distinguishes Streptococcus pyogenes from other non-group A -hemolytic streptococci. On Sheep Blood Agar plate, the strain being tested is streaked with several individual colonies of a pure culture, followed by a disc containing $0.04 \mathrm{U}$ of bacitracin has to be placed. A zone of inhibition surrounding the disc after an overnight incubation at $35^{\circ} \mathrm{C}$ in $5 \% \mathrm{CO} 2$ indicates the strain's susceptibility. ${ }^{14}$

\section{Serologic Test}

Anti-Streptolysin O (ASO) titre is elevated $(>200 \mathrm{IU} / \mathrm{ml})$ in most of the streptococcal infections except pyoderma and PSGN(Post Streptococcal Glomerulo Nephritis). ${ }^{8}$ This titre reflect the past and non-present immunological events. Thus, they are not be used to determine whether an individual with GAS and pharyngitis is actually infected only with pharynx or simply a streptococcal carrier. Demonstration of a significant or fourfold increase of the titer in paired serum samples which was taken 7 to 14 days apart will specify an acute or ongoing infection. ${ }^{12}$

Several nucleases produced by $S$. pyogenes are important for bacterial escape from neutrophil extracellular traps. ${ }^{20}$ The immunologic host response to DNase $B$ is the most consistent of the four streptococcal deoxyribonucleases (DNases). DNase B titers starts to appear in two weeks only after onset of infection and may take six to eight weeks to reach the maximum titer value. Moreover, $80-85 \%$ of RF patients will have elevated ASO titers, additional DNase titers may be beneficial. ${ }^{21}$

\section{Antibiotic resistance testing}

Penicillin is commonly considered as the drug of choice for treating S.pyogenes. $10 \%$ of patients showed suspected or confirmed allergies to Penicillin. For this reason, macrolides were considered as an alternative treatment. For macrolides, susceptibility testing has to be performed using erythromycin because susceptibility and resistance of clarithromycin, dirithromycin and azithromycin can also be predicted by testing erythromycin. Currently, resistance testing will often done by automated systems which provide ready-made antibiotic panels for various bacterial species. ${ }^{14}$

\section{Jones Criteria}

Patients with sufficient symptoms, signs and laboratory findings are confirmed to have ARF to fulfill the Jones criteria. ${ }^{4}$ ARF confirmation requires the patient has to meet both one or two major criteria and two minor criteria. This criteria which was last revised in the year of 2015 guided by the different disease burden around the world and it also increases the role of echocardiography. It was revised based on the risk stratification, subclinical carditis as a major criteria and joint manifestations. It is classified based on the incidence of local disease into two sets of criteria as low risk population with the cut off of $\leq 2$ per 100,000 school childrens in the age group ranges from 5-14 years and moderate/ high risk population in all age prevalence of RHD with the cut off of $\leq 1$ per 100,000 per year. Heart involvement remains the most important condition among the other manifestations as it results in the damage of the valves (RHD) and it also considered as the major cause of acquired heart disease in the developing world. Except carditis, other manifestations will resolve without leaving any permanent consequences. ${ }^{22,23}$

$1^{\text {st }}$ degree atrio ventricular block i.e., prolonged $P R$ interval is the important finding in ECG incase of carditis and echocardiography has become an important tool after the inclusion of subclinical carditis as a major criteria. Echocardiography is not only useful in differentiating pathologic and physiologic regurgitation but also assessing the severity of the lesions. Difficulties in obtaining bacteriological diagnosis as well as in the documentation of fever, any children or adolescents presenting with major joint manifestation and an elevated inflammatory 
markers regardless of previous fever are subjected to echocardiography. ${ }^{24}$

Most of the ARF cases gets missed or does not present in the initial stage rather it has been developed into advanced RHD condition. This revised criteria will be helpful especially to the low risk population as it is challenging because of limited access to primary health care, diagnosis of streptococcal disease. In addition to this revised criteria, diagnosis still relies on clinical diagnostic algorithm. There remains a need to use immunophenotyping and genotyping techniques to understand the pathogenesis, biomarkers to identify the severity and the stage of the illness. ${ }^{25}$ Vaccine Strategies

Vaccines are needed to reduce the burden of GAS. A major GAS virulence determinant is the surface $\mathrm{M}$ protein. $\mathrm{N}$-terminal regions in $\mathrm{M}$ protein consists of higher amount of amino acid residues which results in an antigenic diversity (emm types) and these were formulated with the recombinant proteins to produce the vaccine candidates of GAS: (i) 6-valent, (ii) 26-valent, and (iii) 30-valent. Later, J8 $\mathrm{M}$ protein was formulated together with another vaccine candidate from the $\mathrm{C}$ - terminal region. Importantly, many regions have limited epidemiological data, making vaccine coverage estimates imprecise. ${ }^{25,26} \mathrm{~A}$ better understanding of infection, immunity and colonization especially in multiple syndromes like pharyngitis, impetigo, skin and colonization of the pharynx and also about the diverse geographical locations will help in the successful development of $M$ protein based vaccines. Additional studies evaluating different vaccine candidates which also includes other than the $\mathrm{M}$ protein, could also be conducted with a focus on integration with both pharyngitis and impetigo human infection models. ${ }^{27}$ World Health Assembly (WHA) supported the development of vaccine for GAS in 2018 as a part of renewed effort to combat ARF and RHD. A global study of 2083 GAS genomes led to the development of global atlas of vaccine candidate antigens. GAS throat infection based human challenge model has established and it may enhance the development of vaccine. ${ }^{26}$

Distribution of 170 emm types and 750 subtypes will differ based on the regions and countries. Due of this reason, typing in different regions is considered essential for the epidemiological surveillance of the disease. ${ }^{28,29}$

Apart from emm typing, $T$ antigen based vaccines are also studied. T-antigen has a multimeric nature which produces the pilus fibre, GAS pilus has the ability to extend from the cell surface when compared with other GAS proteins thereby penetrating the capsule of hyaluronic acid. Because of its nature of easiest accessibility to the immune system, it makes an appealing vaccine target. TeeVax1-3 has broad coverage against a panel of 21 T-antigens. Because of cross-reactive epitopes, antibody concentrations in serum to the specific T-antigens were higher in the TeeVax1-3 combination than in the corresponding single TeeVax. It clearly demonstrates the possibility of developing GAS vaccine that is broadly protective by targeting pili. $^{30}$

GAS is an iron-deficient bacterium which primarily relies on heme-iron to meet its requirements and survival in the host is dependent on the proteins which is involved in heme capture and heme import. Native human monoclonal antibody TRL186, helps mice survival in therapeutic and prophylactic mouse models after the intraperitoneal challenge with GAS strain (invasive). It is the first monoclonal antibody which increases the survival rate in mouse model and it is a proof of concept for immunotherapy using monoclonal antibodies that target GAS in heme uptake process. ${ }^{31}$

After a single oral immunization, mucosal and systemic antibodies were produces by the induction of peptide-polymer conjugate. While the antibodies produced after a second immunisation were opsonic, more challenging experiments on GAS will further be needed to confirm the vaccine efficacy. ${ }^{32}$

\section{CONCLUSION}

Rheumatic heart disease is a preventable cardiovascular disease. Improving living conditions, community awareness, educating the patients about the significance of antibiotic prophylaxis, proper maintenance of ARF/RHD registry, monitoring the supply of penicillin to the respected individual, training of front line healthcare providers in the diagnosis and management of Streptococcal disease is the most efficient way 
of reducing ARF and RHD burdens. Preventing ARF recurrence through long-term antibiotic prophylaxis helps to lower RHD progression and severity. Molecular techniques, mechanical intervention techniques and advancement in the diagnosis of established RHD are also continuing to evolve, with the improving results. Ongoing vaccine development is still challenging for the GAS research community, it will make a positive and lasting impact on the peoples globally.

\section{ACKNOWLEDGMENTS}

The authors would like to thank Chettinad Academy of Research and Education, Kelambakkam, India for funding and support.

\section{CONFLICT OF INTEREST}

The authors declare that there is no conflict of interest.

\section{AUTHORS' CONTRIBUTION}

All authors listed have made a substantial, direct and intellectual contribution to the work, and approved it for publication.

\section{FUNDING}

None.

\section{DATA AVAILABILITY}

All datasets generated or analysed during this study are included in the manuscript.

\section{ETHICS STATEMENT}

The study was conducted according to the guidelines of Institutional Human ethical committee (619/IHEC/12-19) - Chettinad Academy of Research and Education, Kelambakkam, India.

\section{REFERENCES}

1. Hand RM, Snelling TL, Carapetis JR. Group A Streptococcus. Hunter's Tropical Medicine and Emerging Infectious Diseases. 2020:429-438. doi: 10.1016/B978-0-323-55512-8.00040-5

2. Cunningham MW. Pathogenesis of group Astreptococcal infections. Clin Microbiol Rev. 2000;13(3):470-511. doi: 10.1128/CMR.13.3.470

3. Smoot LM, McCormick JK, Smoot JC, et al. Characterization of two novel pyrogenic toxin superantigens made by an acute rheumatic fever clone of Streptococcus pyogenes associated with multiple disease outbreaks. Infection and immunity. 2002;70(12):7095-7104. doi: 10.1128/IAI.70.12.7095-

\subsection{2}

4. Eranki A. Acute Rheumatic Fever. Introduction to Clinical Infectious Diseases. Springer, Cham. 2019:125131. doi: 10.1007/978-3-319-91080-2_12

5. Sika-Paotonu D, Beaton A, Raghu A, et al. Acute rheumatic fever and rheumatic heart disease. Ferretti $\mathrm{JJ}$, Stevens DL, Fischetti VA In Streptococcus pyogenes: Basic Biology to Clinical Manifestations. 2017. https:// www.ncbi.nlm.nih.gov/books/NBK425394/

6. Karthikeyan G. Rheumatic Heart Disease in India: Declining, but not fast enough. Natl Med J India. 2017;30(5):247-248.doi: 10.4103/0970-258X.234389

7. Patterson MJ. Streptococcus. Baron S In Medical Microbiology. $4^{\text {th }}$ edition. University of Texas Medical Branch at Galveston. 1996. https://www.ncbi.nlm.nih. gov/books/NBK7611/

8. Sastry AS, Bhat S. Essentials of medical microbiology. Jaypee Brothers, Medical Publishers Pvt. Limited; 2018.

9. Walker MJ, Barnett TC, McArthur JD, et al. Disease manifestations and pathogenic mechanisms of group A Streptococcus. Clin Microbiol Rev. 2014;27(2):264301. doi: 10.1128/CMR.00101-13

10. Webb RH, Grant C, Harnden A. Acute rheumatic fever. BMJ. 2015;351:h3443doi: 10.1136/bmj.h3443

11. Galvin JE, Hemric ME, Ward K, Cunningham MW. Cytotoxic $\mathrm{mAb}$ from rheumatic carditis recognizes heart valves and laminin. J Clin Invest. 2000;106(2):217224. doi: $10.1172 / \mathrm{JCl} 132$

12. Brahmadathan KN, Gladstone P. Microbiological diagnosis of streptococcal pharyngitis: lacunae and their implications. Indian J Med Microbiol. 2006;24(2):92-96. doi: 10.1016/S0255-0857(21)024051

13. Bisno AL, Gerber MA, Gwaltney Jr JM, Kaplan EL, Schwartz RH. Diagnosis and management of group A streptococcal pharyngitis: a practice guideline. Clin Infect Dis. 1997;25(3):574-583. doi: 10.1086/513768

14. Spellerberg B, Brandt C Laboratory diagnosis of Streptococcus pyogenes (group A streptococci). In Ferretti JJ, Stevens DL, Fischetti VA. Streptococcus pyogenes: Basic Biology to Clinical Manifestations. 2016. https://www.ncbi.nlm.nih.gov/books/ NBK343617/

15. Shulman ST, Bisno AL, Clegg HW, et al. Clinical practice guideline for the diagnosis and management of group A streptococcal pharyngitis: 2012 update by the Infectious Diseases Society of America. Clin Infect Dis. 2012;55(10):e86-e102. doi: 10.1093/cid/cis629

16. Bisno AL. Acute pharyngitis. $N$ Engl J Med. 2001;344(3):205-211.doi: 10.1056/ NEJM200101183440308

17. Kaufhold A, Lutticken R, Schwien U. Few-minutes tests for the identification of group $A$ streptococci and enterococci with chromogenic substrates. Zentralbl Bakteriol. 1989;272(2):191-195. doi: 10.1016/S09348840(89)80006-4

18. Coleman DJ, McGhie D, Tebbutt GM. Further studies on the reliability of the bacitracin inhibition test for the presumptive identification of Lancefield group A streptococci. J Clin Pathol. 1977;30(5):421-426 . doi: 10.1136/jcp.30.5.421

19. Murray PR, Wold AD, Hall MM, Washington II 
JA. Bacitracin differentiation for presumptive identification of group $A$-hemolytic streptococci: Comparison of primary and purified plate testing. J Pediatr. 1976;89(4):576-579.doi: 10.1016/S00223476(76)80389-7

20. Sumby P, Barbian KD, Gardner DJ, et al. Extracellular deoxyribonuclease made by group A Streptococcus assists pathogenesis by enhancing evasion of the innate immune response. Proc Natl Acad Sci U S A. 2005;102(5):1679-1684. doi: 10.1073/ pnas. 0406641102

21. Shet A, Kaplan EL. Clinical use and interpretation of group A streptococcal antibody tests: a practical approach for the pediatrician or primary care physician. Pediatr Infect Dis J. 2002;21(5):420-426. doi: 10.1097/00006454-200205000-00014

22. Marino A, Cimaz R, Pelagatti MA, et al. Acute rheumatic fever: where do we stand? An epidemiological study in northern Italy. Front Med. 2021;8:621668. doi: 10.3389/fmed.2021.621668

23. Figueroa FE, Fernandez MS, Valdes $\mathrm{P}$, et al. Prospective comparison of clinical and echocardiographic diagnosis of rheumatic carditis: long term follow up of patients with subclinical disease. Heart. 2001;85(4):407-410. doi: 10.1136/heart.85.4.407

24. Arvind B, Ramakrishnan S. Rheumatic fever and rheumatic heart disease in children. Indian J Pediatr. 2020;87(4):305-311. doi: 10.1007/s12098-019-031287

25. Beaton A, Carapetis J. The 2015 revision of the Jones criteria for the diagnosis of acute rheumatic fever: implications for practice in low-income and middleincome countries. Heart Asia. 2015;7(2):7-11. doi: 10.1136/heartasia-2015-010648

26. Jabang S, Erhart A, Darboe S, et al. Molecular Epidemiology of Group A Streptococcus Infections in The Gambia. Vaccines. 2021;9(2):124. doi: 10.3390/ vaccines9020124

27. Beall B, Van Beneden C. Challenges to vaccine development: the diversity of Group A streptococcal strains among varied climates and global regions. J Infect Dis. 2020;221(9):1394-1397. doi: 10.1093/ infdis/jiz617

28. Frost HR, Davies MR, Velusamy S, et al. Updated emm-typing protocol for Streptococcus pyogenes. Clin Microbiol Infect. 2020;26(7):946.e5-946e8.doi: 10.1016/j.cmi.2020.02.026

29. Himri S, Oumokhtar B, Atmani S, et al. The Place of Group A Streptococci in Moroccan Children with Pharyngitis and Emm Type Distribution. Arch Pediatr Infect Dis. 2021;9(4):e111172. doi: 10.5812/ pedinfect.111172

30. Loh JM, Rivera-Hernandez T, McGregor R, et al. A multivalent T-antigen-based vaccine for Group A Streptococcus. Sci Rep. 2021;11(1):4353.d oi: 10.1038/ s41598-021-83673-4

31. Chatterjee N, Huang YS, Lyles KV, et al. Native Human Antibody to Shr Promotes Mice Survival After Intraperitoneal Challenge With Invasive Group A Streptococcus. J Infect Dis. 2021;223(8):1367-1375. doi: 10.1093/infdis/jiaa540

32. Faruck MO, Zhao L, Hussein WM, et al. PolyacrylatePeptide Antigen Conjugate as a Single-Dose Oral Vaccine against Group A Streptococcus. Vaccines. 2020;8(1):23. doi: 10.3390/vaccines 8010023 